\title{
Suppression of Exponential Electronic Decay in a Charged Environment
}

\author{
Vitali Averbukh, ${ }^{1, *}$ Ulf Saalmann, ${ }^{1,2}$ and Jan Michael Rost ${ }^{1,2}$ \\ ${ }^{1}$ Max Planck Institute for the Physics of Complex Systems, Nöthnitzer Strasse 38, D-01187 Dresden, Germany \\ ${ }^{2}$ Max Planck Advanced Study Group at the Center for Free-Electron Laser Science, Luruper Chaussee 149, 22761 Hamburg, Germany
}

(Received 28 February 2010; published 7 June 2010)

\begin{abstract}
Inner-shell ionization of atoms and molecules leads to the creation of highly excited ionic states that often decay by electron emission. The dynamics of the decay is usually assumed to be exponential and the process is characterized by a decay rate. Here we show that in a multiply ionized cluster created by interaction with a high-intensity free-electron laser (FEL) radiation, trapping of the emitted electron by the neighboring ions changes the character of the decay dynamics qualitatively to the extent that it can become oscillatory instead of exponential. Implications of the predicted effect on Coster-Kronig and interatomic Coulombic decay processes induced by FELs are investigated.
\end{abstract}

$\mathrm{X}$-ray free-electron lasers (XFELs) [1], are expected to open a new era in the study of radiation-matter interaction. A unique combination of high photon energies with the high intensities provided by the XFELs is anticipated to lead to a completely new dynamical regime, changing many of our basic perceptions in such areas as atomic and molecular physics, plasma physics, structural biology, etc. Central to the proposed applications of the intense XFELs to structural biology [2] is the issue of radiation damage [3]. The question to be asked, for instance in single-molecule diffractive imaging, is what happens first: the formation of a useful diffraction picture or the destruction of the macromolecule? The radiation damage is a result of a complex set of physical processes, all leading to accumulation of positive charge and the ensuing Coulombic explosion of the system. A major role in the positive charge accumulation is played by the electronic decay processes. Among them, the Auger effect [4], i.e., the decay of a core-ionized state of an atom or a molecule by electron emission, is the most familiar one. Apart from the direct contribution to the multiple ionization of the core-ionized system, the Auger effect is responsible for cascades of electron impact ionization processes initiated by inelastic scattering of Auger electrons on the surrounding atoms [5]. Hence, understanding the dynamics of Auger decay is essential for an accurate estimate of the charge accumulation in molecule-XFEL interaction and eventually for the design of the XFEL pulses capable of extracting valuable structural information [6].

The dynamics of electronic decay in isolated atomic and small molecular systems ionized by low-intensity (e.g., synchrotron) radiation is understood fairly well [4]. Usually, the inner-shell ionization event and the decay of the resulting vacancy state can be treated separately, with the latter described as a nonradiative transition. Fano theory of resonances [7] provides a particularly useful formalism for the theoretical treatment of the decay process. Within this formalism, the nonradiative transition occurs due to coupling between the artificially constructed boundlike state, $\Phi$, describing the inner-shell ionized system with the energy above the double ionization threshold, and the continuum states of the same energy, $\chi_{\beta, \epsilon_{\beta}}$ describing the doubly ionized system in its $\beta$ th state with the energy $E_{\beta}$ and the emitted Auger electron with kinetic energy $\epsilon_{\beta}$. In the Fano approach, the continuum states $\chi_{\beta, \epsilon_{\beta}}$ are constructed in such a way that, to a good approximation, they are not coupled by the full Hamiltonian $\hat{H}$ :

$$
\left\langle\chi_{\beta^{\prime}, \epsilon^{\prime}}|\hat{H}|_{\beta, \epsilon}\right\rangle \approx\left(E_{\beta}+\epsilon\right) \delta_{\beta^{\prime}, \beta} \delta\left(E_{\beta^{\prime}}+\epsilon^{\prime}-E_{\beta}-\epsilon\right) .
$$

It can be shown [7] that the boundlike state $\Phi$, once prepared, will decay approximately exponentially in time,

$$
|\langle\Phi \mid \Psi(t)\rangle|^{2}=e^{-\Gamma t / \hbar}, \quad \Psi(t=0)=\Phi,
$$

where $\Psi(t)$ is the full wave function of the system and the decay rate, $\Gamma / \hbar$, is given through

$$
\Gamma=\sum_{\beta=1}^{N_{c}} \Gamma_{\beta}=2 \pi \sum_{\beta=1}^{N_{c}}\left|\left\langle\Phi\left|\hat{H}-E_{r}\right| \chi_{\beta, \epsilon_{\beta}}\right\rangle\right|^{2},
$$

where $N_{c}$ is the number of bound states of the doubly ionized system being energetically lower than the innershell-ionized state $\Phi$, or in other words, the number of open decay channels, and $E_{r}$ is the energy of the decaying state.

In the present work, we show that the well-known picture of the electronic decay dynamics outlined above can change qualitatively once the decay is induced by a highintensity XFEL interaction with a polyatomic system. The reason for the predicted change is the ability of the XFEL radiation to promote the target system into a highly charged state well within the pulse duration [8]. As a result, electronic decay of an atomic inner-shell vacancy induced by an XFEL pulse interaction with a molecule or cluster will typically occur in a highly charged environment. 
Under such conditions, the kinetic energy of the Auger electron may not suffice for leaving the system and the electron will be trapped by the field of the positive charges surrounding the inner-shell ionized atom [9]. On a more formal level, the final states of the decay, $\chi_{\beta, \epsilon}$, around the resonance energy $E_{r}$, will no longer form a continuum, but will rather turn into a quasicontinuum of closely spaced bound states. An electron occupying one of such states will be delocalized across the whole system, yet, will not be able to leave it. With discrete final states, however, one cannot expect the familiar notion of the exponential decay to remain valid and the very basics of our understanding of electronic decay dynamics should be reconsidered. Indeed, a trapped (e.g., Auger) electron can participate in an "inverse Auger" process, i.e., it can fill one of the vacancies created by the Auger transition, giving a core electron enough energy to be promoted to another vacancy of the Auger final state. Therefore, the survival probability of the core hole state [see Eq. (2)] can exhibit oscillatory rather than exponential character. This situation is formally similar to the one encountered in the nonradiative decay of excited electronic molecular states due to vibronic coupling. For the idealized Bixon-Jortner (BJ) model [10], one can show that the exponential decay persists for the times of the order of inverse quasicontinuum spacing $\hbar / \varepsilon$. However, the BJ model could be hardly applicable to the complicated many-electron states involved in the electronic decay. The essential open question is therefore: Can electronic decay processes in a highly charged environment be characterized, even approximately, by exponential dynamics?

We would like to start clarifying the character of the electronic decay dynamics in a multiply charged system by constructing a simple, but realistic model based on the $L_{1}-L_{2,3} M$ Coster-Kronig (CK) decay in argon (see Ref. [11] and references therein). Consider an Ar cluster, e.g. $\mathrm{Ar}_{13}$, interacting with an intense XFEL radiation. Neutral $\mathrm{Ar}_{13}$ in its ground state has an icosahedral $\left(I_{h}\right)$ equilibrium geometry with the central $\mathrm{Ar}$ and 12 symmetry-equivalent peripheral $\mathrm{Ar}$ atoms at a distance of $R_{\text {eq }}=6.96$ a.u. [12]. Simulations of $\mathrm{Ar}_{13}$-XFEL interaction [8] show that one could easily reach a situation where all the Ar atoms in the cluster are singly ionized well before the cluster geometry suffers a significant distortion. Imagine, for simplicity, that the central atom of $\mathrm{Ar}_{13}$ is ionized in its $2 s$ subshell, while the 12 surrounding argons bear either $2 p$ or outer-shell vacancies. Such a distribution of vacancies among the electronic shells is not implausible given that the photoionization cross sections of the outer shell and $2 p$ electrons of Ar are higher than that of the $2 s$ electron [13]. With the lifetime of about 0.3 fs [11], CK decay of the $2 s$ vacancy is more than an order of magnitude faster than Auger decay of $\left(2 p^{-1}\right) \mathrm{Ar}^{+}[11,14]$. The time scale for the second ionization of one of the argons in the case of a long ( $\sim 100 \mathrm{fs})$ XFEL pulse is controlled by the pulse intensity and can be in the range between 10 and
$100 \mathrm{fs}$ [8]. For these reasons, we assume the vacancy states of the $12 \mathrm{Ar}$ atoms surrounding the $2 s$-ionized one to be stationary on the time scale of the CK process. If, nevertheless, the Auger decay or a second photoionization event occurs, it would effectively increase the positive charge of the cluster and lead to a stronger trapping of the would-be CK electron.

The kinetic energies of the CK electrons generated by the $L_{1}-L_{2,3} M$ decay in isolated $\left(2 s^{-1}\right) \mathrm{Ar}^{+}$vary between about 29 and $47 \mathrm{eV}$ [11]. In a multiply ionized $\mathrm{Ar}_{13}$ cluster, the energies of the corresponding final doubly ionized states of the central Ar will be elevated in the simple point charge approximation by $\Delta E=12 / R_{\mathrm{eq}}$. This turns out to be just enough to put all the $\left(2 p^{-1} 3 l^{-1}\right) \mathrm{Ar}^{2+}\left(\mathrm{Ar}^{+}\right)_{12}$ states of the cluster above the energy of the initial $2 s$ vacancy. Hence, as long as the ionized cluster does not expand, the CK electron which left the central argon will always be bound to the cluster as a whole. In what follows, we would like to study the implication of the CK electron trapping in $\left(2 s^{-1}\right) \mathrm{Ar}^{+}\left(\mathrm{Ar}^{+}\right)_{12}$ on the dynamics of the electronic decay process.

Following Fano [7], we describe the nonradiative decay process in $\left(2 s^{-1}\right) \mathrm{Ar}^{+}\left(\mathrm{Ar}^{+}\right)_{12}$ as a time evolution of the boundlike state, $\Phi$, corresponding to the initial $2 s$ vacancy, coupled to a series of discrete states $\chi_{n}$, representing the doubly ionized $\left(2 p^{-1} 3 l^{-1}\right)$ central atom and a CK electron delocalized across the $\mathrm{Ar}^{2+}\left(\mathrm{Ar}^{+}\right)_{12}$ cluster. Since our model considers the vacancies on the peripheral argons as spectators, at least on the time scale of the CK transition, we substitute them by point charges, i.e., we consider the $\left(2 s^{-1}\right) \mathrm{Ar}^{+}\left(\mathrm{H}^{+}\right)_{12}$ system in the equilibrium geometry of the neutral $\mathrm{Ar}_{13}$. This allows us to represent the manyelectron states of multiply ionized cluster using the $a b$ initio methods developed for characterization of the singly ionized closed-shell systems. More specifically, we employ the extended second order algebraic diagrammatic construction scheme $[\operatorname{ADC}(2) x][15]$ in the so-called intermediate state representation (ISR) [16]. Within the ISRADC(2)x scheme, the many-electron Hamiltonian of a singly ionized system is represented in the basis of the intermediate states of one-hole (1h), $\tilde{\Psi}_{i}$, and two-hole-one-particle $(2 h-1 p), \tilde{\Psi}_{j k}^{a}$, nature. Here, the indices $i, j, k$ run over the occupied orbitals, e.g., Ar $2 s$, while the index $a$ corresponds to an unoccupied orbital of the $\operatorname{Ar}\left(\mathrm{H}^{+}\right)_{12}$ system prior to $2 s$ ionization. An energy-based intermediate state selection scheme [17], allows us to sort the $2 h-1 p$ states into two subspaces: those, which contribute to the ADC(2)x expansion for the boundlike initial state, $\Phi$, and those which enter $\operatorname{ADC}(2) x$ expansion of the final states, $\chi_{\epsilon_{n}}$. The initial state is given by

$$
\Phi=a_{2 s} \tilde{\Psi}_{2 s}+\sum_{j k a} b_{j k}^{a} \tilde{\Psi}_{j k}^{a},
$$

where the possible pairs of occupied orbital indices, $j, k$, are restricted to the doubly ionized configurations with energies higher than that of $\Psi_{2 s}$. The final states are given by 


$$
\chi_{n}=\sum_{j^{\prime} k^{\prime} a^{\prime}} c_{j^{\prime} k^{\prime}}^{a^{\prime}(n)} \tilde{\Psi}_{j^{\prime} k^{\prime}}^{a^{\prime}}
$$

where the possible pairs of occupied orbital indices, $j^{\prime}, k^{\prime}$, are restricted to doubly ionized configurations with energies lower than that of $\Psi_{2 s}$, i.e., to the open channels of the CK transition in an isolated $\operatorname{Ar}$ [18]. The expansion coefficients $a_{2 s}, b_{j k}^{a}, c_{j^{\prime} k^{\prime}}^{a^{\prime}(n)}$ and the corresponding eigenenergies, $E_{\Phi}, E_{n}$, are obtained by diagonalization of the $\operatorname{ADC}(2) \mathrm{x}$ Hamiltonian matrices constructed in the basis of the intermediate states of the appropriate (initial state of final state) subspace. With the initial and final states of the nonradiative transition specified, we obtain the evolution of the initial state by propagating it in time under the Fano type Hamiltonian, $\hat{H}$, built in the basis of $\left\{\Phi, \chi_{n}\right\}$ :

$\Psi(t)=e^{-i \hat{H} t / \hbar} \Phi$,

$$
\hat{H}=|\Phi\rangle E_{\Phi}\left\langle\Phi\left|+\sum_{n}\right| \chi_{n}\right\rangle E_{n}\left\langle\chi_{n}\right|+\left(|\Phi\rangle \gamma_{n}\left\langle\chi_{n}\right|+\text { c.c. }\right),
$$

where the initial-final coupling matrix elements are given by $\gamma_{n}=\left\langle\Phi|\hat{H}| \chi_{n}\right\rangle$.

We construct the initial and the final states of the CK decay in $\left(2 s^{-1}\right) \mathrm{Ar}^{+}\left(\mathrm{H}^{+}\right)_{12}$ according to Eqs. (4) and (5) using the restricted Hartree-Fock (RHF) solution of the $\operatorname{Ar}\left(\mathrm{H}^{+}\right)_{12}$ electronic ground state. The RHF solution has been obtained using the MOLCAS6 quantum chemical program package [19] within the uncontracted cc-pCVQZ Gaussian basis [20] augmented by 6 spdf diffuse Kaufmann-Baumeister-Jungen (KBJ) Gaussians [21] for Ar and uncontracted cc-pVQZ [20] augmented by $10 s p$ diffuse KBJ Gaussians for $\mathrm{H}^{+}$. The survival probability of the $2 s$ vacancy state of the central Ar atom in the model $\mathrm{Ar}_{13}$ cluster obtained using the Eqs. (2) and (6) [see Fig. 1(a)] shows a roughly exponential dynamics on the short time scale. During the initial $0.7 \mathrm{fs}$, the population of the $2 s$ vacancy state exhibits oscillations superimposed over an exponential decay with the mean rate being very close to the one of the $L_{1}-L_{2,3} M$ transition in an isolated Ar. A striking change of behavior occurs, however, at $t>0.7 \mathrm{fs}$, where even the approximately exponential decay pattern is suppressed completely by an oscillatory one. During this phase of the time evolution, the $2 s$ vacancy population rises roughly from $1 \%$ to $10 \%$ in a clear manifestation of a partial "inverse Auger" transition and then falls back. At later times, the calculated survival probability keeps oscillating within a similar range. Our assumption that surrounding vacancies are only spectators limits, however, the validity of the present calculation to $\tau<\tau_{L_{2,3}-M M}=3.7$ fs. Another insight into the mechanism of the exponential decay suppression comes from the examination of $\left\langle\Psi(t)\left|r^{2}\right| \Psi(t)\right\rangle$. In Fig. 1 one can easily notice that the plots of the spatial extent of $\Psi(t)$ and of the survival probability of the $2 s$ vacancy state behave as mirror images of each other. This suggests that the Auger electron wave packet emitted by the central ion is reflected back by the charged cluster potential, leading to peaks of $\left\langle r^{2}\right\rangle(t)$ [Fig. 1(b)]. Propagating back towards the
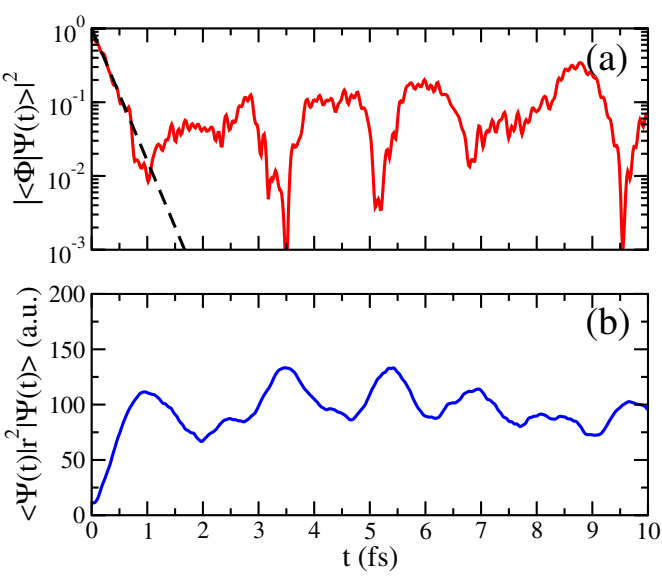

FIG. 1 (color online). (a) Solid line-survival probability of the $2 s$ vacancy in $\operatorname{Ar}^{+}\left(\mathrm{H}^{+}\right)_{12}$ plotted on the logarithmic scale. Dashed line-same for the exponential decay of the $2 s$ vacancy in an isolated $\mathrm{Ar}^{+}$. (b) Time dependence of the $r^{2}$ expectation value measuring the spatial extent of $\Psi(t)$.

central atom, the Auger electron partly recombines in an inverse Auger transition leading to the partial revivals of $|\langle\Phi \mid \Psi(t)\rangle|^{2}$ [Fig. 1(a)].

In the particular example discussed above, the CK electronic transition is so fast that the decay process is almost completed before the emitted electron "discovers" the finite size of the system and the oscillations set in. It would be, thus, interesting to follow the suppression of the exponential decay in the opposite extreme, namely, under conditions of slow nonradiative transition. To this end, we consider an interatomic Coulombic decay (ICD) process [22] following $\mathrm{Ne} 2 s$ ionization in the octahedral $\mathrm{Ne}_{6}$ cluster [23] in which we assume another neon atom to be $2 p$ ionized [24]. The low kinetic energy of the Ne ICD electrons (about $1.2 \mathrm{eV}$ ) guarantees that the ICD electron trapping can be achieved even by a single additional charge in the $\mathrm{Ne}_{6}$ cluster. As in the case of our $\mathrm{Ar}_{13}$ model, we substitute the atom bearing the spectator vacancy by a point charge. In our Ne ICD calculations, we use the ccCPVQZ basis set [20] augmented 6 spd KBJ Gaussians [21] for on Ne and cc-PVQZ [20] augmented by $6 s p d$ KBJ Gaussians for $\mathrm{H}^{+}$. The survival probability of the $\mathrm{Ne} 2 s$ vacancy obtained using Eqs. (2) and (6) is shown in Fig. 2. One easily notices that the exponential character of the slow ICD [more than two order or magnitude slower than $\left(2 s^{-1}\right) \mathrm{Ar}^{+} \mathrm{CK}$ decay] is completely suppressed from the beginning. Simple classical considerations suggest that later, say, after about $70 \mathrm{fs}$, the geometry of the doubly ionized cluster will be distorted noticeably (by about 10\%) due to the repulsive nuclear dynamics. Thus, the subsequent revivals (not shown here) will be affected by the nuclear motion which is not taken into account by the present model. Remarkably, suppression of the exponential character of the ICD leads here to a faster loss of the decaying state population than it would be the case in the absence of the additional positive charge. 


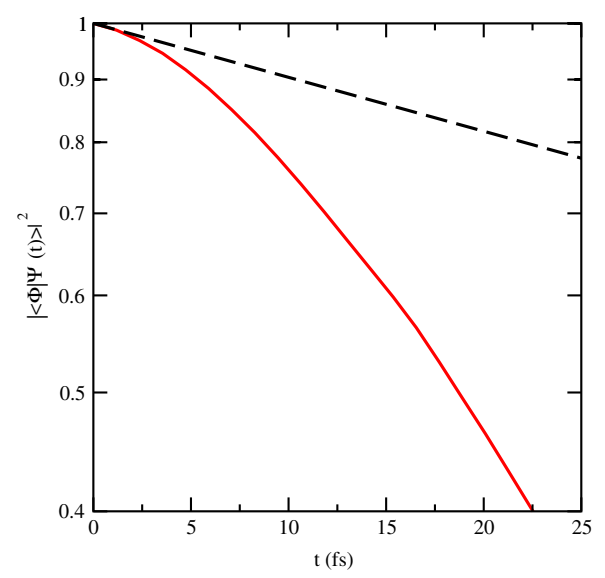

FIG. 2 (color online). Survival probability of a $2 s$ vacancy in $\mathrm{Ne}_{5} \mathrm{H}^{+}$plotted on a logarithmic scale. Solid line-FanoADC(2)x calculation, Eqs. (4) and (5), dashed line-exponential ICD in the $\left(2 s^{-1}\right) \mathrm{Ne}^{+} \mathrm{Ne}_{4}$ taken as a fragment of the $\mathrm{Ne}_{6}$ structure [23]. The ICD width was calculated using the FanoADC(2)x approach of Ref. [17].

In conclusion, we have shown that the dynamics of the electronic decay of inner-shell vacancies in a charged environment, such as created by interaction of a cluster with a high-intensity FEL radiation, can be qualitatively different from the one induced by a low-intensity source. If the emitted electrons are slow enough to be trapped by the neighboring charges, the familiar exponential decay will be suppressed by quantum beats between the initial state and the quasicontinuum of discrete final states. Physically, the predicted oscillations correspond to creation of the initial vacancy due to the reflections of the emitted electron by the charged cluster potential and the subsequent inverse Auger transition. If the decay process in the isolated singly charged system is fast on the time scale of the quantum beats, as it is in the case of CK transition in multiply ionized $\mathrm{Ar}_{13}$, the survival probability of the initial vacancy can reach values close to zero before the oscillations set in. In the opposite limit of slow electronic decay the exponential dynamics is suppressed completely.

We expect the new dynamical picture of electronic decay in multiply charged systems to be important for the simulations of the Coulombic explosion of clusters and molecules exposed to XFEL radiation. Indeed, Auger channel closings due to the multiple ionization and the resulting trapping of Auger electrons is a phenomenon characteristic of XFEL-induced dynamics. As we have shown, exponential decay can remain a good approximation for the fast decay processes, but can turn out to be irrelevant for the slow ones. The nonexponential, possibly oscillatory dynamics of electronic decay predicted here can be verified experimentally, both directly and indirectly. A direct observation can be accomplished, for example, through the femto- or attosecond probing of the population of the inner-shell vacancy. A comparison of the experimental results on the XFEL-induced Coulombic explosion with the simulations taking into account the new effect as well as with those neglecting it, could provide an indirect support for the predicted suppression of the exponential dynamics of the electronic decay.

V. A. would like to thank Dr. Albert Stolow for fruitful discussions.

*vitali@mpipks-dresden.mpg.de

[1] J. Feldhaus, J. Arthur, and J. B. Hastings, J. Phys. B 38, S799 (2005)

[2] K. J. Gaffney and H.N. Chapman, Science 316, 1444 (2007).

[3] R. Neutze et al., Nature (London) 406, 752 (2000).

[4] W. Mehlhorn, in Atomic Inner-Shell Physics, edited by B. Crasemann (Plenum, New York, 1985); H. Ågren, A. Cesar, and C.-M. Liegener, Adv. Quantum Chem. 23, 1 (1992).

[5] M. Bergh, N. Tîmneanu, and D. van der Spoel, Phys. Rev. E 70, 051904 (2004).

[6] S. Hau-Riege et al., Phys. Rev. E 71, 061919 (2005).

[7] U. Fano, Phys. Rev. 124, 1866 (1961).

[8] U. Saalmann and J. M. Rost, Phys. Rev. Lett. 89, 143401 (2002).

[9] C. Gnodtke, U. Saalmann, and J. M. Rost, Phys. Rev. A 79, 041201(R) (2009);

[10] M. Bixon and J. Jortner, J. Chem. Phys. 48, 715 (1968).

[11] T. Kylli et al., Phys. Rev. A 59, 4071 (1999).

[12] D. J. Wales et al., The Cambridge Cluster Database, 2007, http://www-wales.ch.cam.ac.uk/CCD.html; A. Cuccoli et al., Phys. Rev. B 47, 14923 (1993).

[13] D. A. Verner et al., At. Data Nucl. Data Tables 55, 233 (1993).

[14] J. C. Fuggle and S.F. Alvarado, Phys. Rev. A 22, 1615 (1980).

[15] J. Schirmer, L. S. Cederbaum, and O. Walter, Phys. Rev. A 28, 1237 (1983); L. S. Cederbaum, in Encyclopedia of Computational Chemistry, edited by P.v. R. Schleyer et al. (Wiley, New York, 1998).

[16] J. Schirmer, Phys. Rev. A 43, 4647 (1991).

[17] V. Averbukh and L. S. Cederbaum, J. Chem. Phys. 123, 204107 (2005).

[18] Here we neglect the contribution of the much slower $L-M M$ Auger process.

[19] K. Andersson et al., MOLCAS Version 6.4 (Lund University, Sweden, 2004).

[20] K. L. Schuchardt et al., J. Chem. Inf. Model. 47, 1045 (2007).

[21] K. Kaufmann, W. Baumeister, and M. Jungen, J. Phys. B 22, 2223 (1989).

[22] L. S. Cederbaum, J. Zobeley, and F. Tarantelli, Phys. Rev. Lett. 79, 4778 (1997).

[23] D. Blume and C. Greene, J. Chem. Phys. 113, 4242 (2000).

[24] See B. Müller and L. S. Cederbaum, J. Chem. Phys. 122, 094305 (2005) for the ICD in the presence of a positive charge and B. Müller, J. Zobeley, and L. S. Cederbaum, J. Chem. Phys. 117, 1085 (2002) for the opposite effect of a negative charge. 